\title{
Endoreversible Four-Reservoir Chemical Potential Transformer with Diffusive Mass Transfer Law
}

\author{
D. XIA ${ }^{a, b}$, L. $\mathrm{CHEN}^{a, *}$ AND F. SUN ${ }^{a}$ \\ ${ }^{a}$ College of Naval Architecture and Power, , Naval University of Engineering, Wuhan 430033, P.R. China \\ b92957 troop, Zhoushan 316000, P R China
}

(Received June 19, 2010; in final form December 15, 2010)

\begin{abstract}
The performance of an isothermal endoreversible four-reservoir chemical potential transformer, in which the mass transfer between the mass reservoir and the working medium obeys diffusive law, is analyzed and optimized in this paper. The relation between the rate of energy pumping and the coefficient of performance of the isothermal chemical potential transformer is derived by using finite-time thermodynamics. Moreover, the optimal operating regions and the influences of some parameters on the performance of the cycle are studied. The results obtained herein can provide some new theoretical guidelines for the optimal design of a class of apparatus such as mass exchangers, as well as electrochemical, photochemical, and solid-state devices, and the fuel pumps for solar-energy conversion systems.
\end{abstract}

PACS: 05.70.Ln, 82.60.Lf

\section{Introduction}

In the recent years, finite-time thermodynamics [1-8] has been applied to the performance study of various thermodynamic cycles and devices. It has also been extended to the cyclic devices driven by mass flow, such as chemical reactions and chemical engines, by many researchers. Heat engines generate work from differences in temperature. Similarly, chemical engines generate work from differences in chemical potential. Chemical potential and mass transfer in chemical engines play the analogous roles of temperature and heat current in heat engines $[6,9-20]$.

An extension of the study of isothermal chemical engines, chemical pumps and chemical potential transformers is to analyze the performance characteristics of isothermal chemical cyclic system operating among four-mass-reservoirs at different chemical potentials. A four-reservoir chemical potential transformer is one new type of these isothermal chemical systems. Just as a four-heat-reservoir heat transformer [21, 22] operating among four heat reservoirs is a direct generalization of heat engines, a four-mass-reservoir chemical potential transformer is a direct generalization of chemical engines. The four-mass-reservoir chemical potential transformer is composed of a two-mass-reservoir isothermal chemical pump [16] driven

\footnotetext{
* corresponding author; e-mail:

lgchenna@yahoo.com, lingenchen@hotmail.com
}

by an isothermal chemical engine [12-15]. The isothermal chemical cyclic system is an analogue to the photosynthetic engine discussed by De Vos [23, 24], which consists of a fuel pump (i.e. chemical pump) driven by a photovoltaic engine. The advantage of this new type of chemical potential transformer is characterized by the capability of high degree of chemical potentials upgrading while simultaneously decreasing environmental pollution. The advantage of this new type model of chemical potential transformer is that it is more closely the true model of a two-mass-reservoir chemical pump driven by a chemical engine than the model of three-mass-reservoir chemical potential transformer model [20].

Xia et al. [25] and Chen et al. [26] established the models of an endoreversible four-reservoir chemical pump cycle [25] and a generalized irreversible four-reservoir chemical pump cycle [26], and studied the performance of four-mass-reservoir chemical pump with linear mass transfer law. Xia et al. [27, 28] established the models of an endoreversible four-reservoir chemical potential transformer cycle [27] and a generalized irreversible four-reservoir chemical potential transformer cycle [28], and studied the performances of four-reservoir chemical potential transformer with linear mass transfer law.

In the analysis and optimization mentioned above [9-20, 25-28], the mass transfer between the mass reservoirs and the chemical engine or chemical pump or chemical potential transformer is always assumed to obey linear mass exchange law, i.e. $\Delta N \propto \Delta \mu$, where $\Delta N$ is the exchanged mass and $\Delta \mu$ is the chemical potential difference. However, another linear mass transfer $(\Delta N \propto \Delta c$, 
where $\Delta c$ is the concentration difference) is more prevalent [29-31]. According to Ref. [20], the chemical potential $\mu$ in ideal mixture which is similar in properties to ideal gases or ideal solutions can be expressed as

$$
\mu(c)=\mu_{0}(P, T)+R T \ln c,
$$

where $c$ is the concentration, $P$ and $T$ are the mixture's absolute temperature and pressure, $R$ is the universal gas constant, and $\mu_{0}(P, T)$ is the chemical potential (known for most of substances).

From Eq. (1), $c=\exp \left(\frac{\mu-\mu_{0}}{R T}\right)$ is obtained. The linear mass transfer law $\Delta N \propto \Delta c$ is equivalent to the common diffusive mass transfer law $\Delta N \propto \Delta[\mu /(R T)]$ [12]. They are different mathematical representations for the same mass transfer manner. However, the mass transfer law $\Delta N \propto \Delta[\mu /(R T)]$ expressed as a function of chemical potential is more convenient for calculation and analysis. In Ref. [12], the diffusive mass transfer is expressed as: $\Delta N \propto \Delta[\mu /(k T)]$ ( $k$ is Boltzmann's constant). The choice of coefficient depends on the phase of the substance in the cycle. In this paper, the expression $\Delta N \propto \Delta[\mu /(k T)]$ is adopted.

Although Refs. [12] obtained numerical results of efficiency at maximum power output of chemical engine, which are governed by diffusive mass transfer, no analytical results have been obtained about the power output and efficiency. So it is necessary to investigate the performance of chemical converters which obey the more general and practical mass transfer law: the diffusive mass transfer law. Recently, Chen et al. [32] and Xia et al. [33, 34] modeled and optimized the performance of endoreversible chemical engines [32], endoreversible two-mass-reservoir chemical pumps [33] and endoreversible three-mass-reservoir chemical potential transformer [34] with the diffusive mass transfer law.

The purpose of this paper is to explore the performance of an endoreversible four-reservoir chemical potential transformer by assuming that the mass transfer between the cyclic working medium and the mass reservoir obeys the diffusive mass transfer law. The characteristic between the rate of energy pumping versus the coefficient of performance (COP) is obtained by numerical calculations.

\section{Chemical potential transformer model}

The schematic diagram of an endoreversible chemical potential transformer operating among four mass reservoirs is shown in Fig. 1 . In the figure, $\mu_{\mathrm{H}}, \mu_{\mathrm{L}}, \mu_{0}$ and $\mu_{\mathrm{M}}$ are, respectively, the chemical potentials of the four reservoirs and they are supposed to be constant and have a relation: $\mu_{0}>\mu_{\mathrm{H}}>\mu_{\mathrm{L}}>\mu_{\mathrm{M}}$; parameters $\mu_{1}, \mu_{2}, \mu_{3}$ and $\mu_{4}$ are, respectively, the chemical potentials of the chemicals involved in the processes in the cyclic working medium. Because of the existence of finite-rate mass transfer, $\mu_{1}, \mu_{2}, \mu_{3}$ and $\mu_{4}$ are, respectively, different from those of the four mass reservoirs. Parameters $\Delta N_{1}$, $\Delta N_{2}, \Delta N_{3}$, and $\Delta N_{4}$ are, respectively, the amounts of mass exchange between the cyclic working medium and the four mass reservoirs at chemical potentials $\mu_{\mathrm{H}}, \mu_{\mathrm{L}}$, $\mu_{0}$, and $\mu_{\mathrm{M}}$ per cycle. Parameters $h_{1}, h_{2}, h_{3}$, and $h_{4}$ are, respectively, the mass-transfer coefficients between the cyclic working medium and the mass reservoirs at chemical potentials $\mu_{\mathrm{H}}, \mu_{\mathrm{L}}, \mu_{0}$, and $\mu_{\mathrm{M}}$.

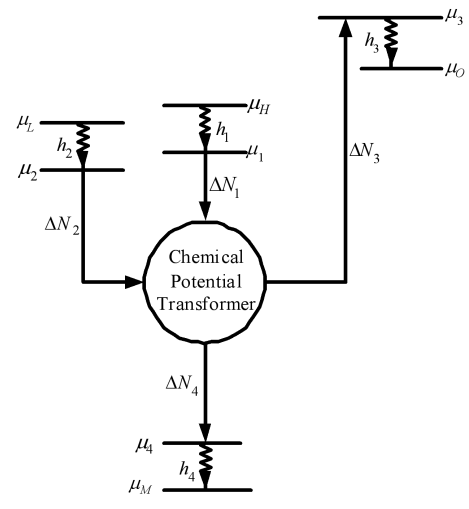

Fig. 1. The model of an isothermal endoreversible four-reservoir chemical potential transformer.

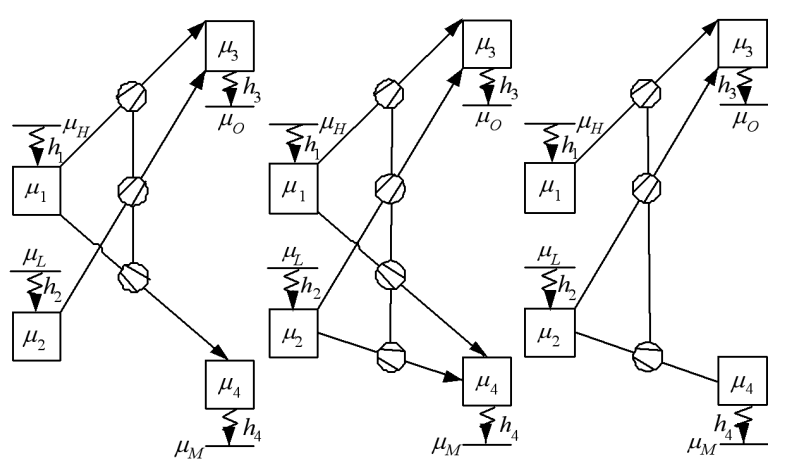

Fig. 2. The possible flow charts of the isothermal endoreversible four-reservoir chemical potential transformer model.

According to Refs. [35, 36], the endoreversible chemical potential transformer has similar flow charts as an absorption heat transformer, as shown in Fig. 2. The imagined chemical engine transfers mass from one or both of the chemical potential levels $\mu_{1}$ and $\mu_{2}$ to the chemical potential level $\mu_{4}$. On the other hand, the imagined chemical pump transfers mass from one or both of the chemical potential levels $\mu_{1}$ and $\mu_{2}$ to the chemical potential level $\mu_{3}$. The different mass transport paths are entirely chemical-potential-dependent. Finally, parameters $t_{1}, t_{2}, t_{3}$ and $t_{4}$ are the corresponding times spent on the four mass transfer processes by the working fluid. Besides the four mass transfer processes between the cyclic working medium and the mass reservoirs, there also exist other additional branches of the cycle that connect the four mass transfer processes. The additional branches do not have mass transfer so that the times spent on the connecting branches without mass transfer are neglected. Therefore, the cyclic period $\tau$ of the chemical potential 
transformer equals, approximately, the sum of $t_{1}, t_{2}, t_{3}$ and $t_{4}$, i.e.

$$
\tau=t_{1}+t_{2}+t_{3}+t_{4} \text {. }
$$

It is assumed that the mass exchange obeys the diffusive mass transfer law of nonlinear irreversible thermodynamics [12], i.e.

$$
\begin{aligned}
& \Delta N_{1}=h_{1}\left(\exp \frac{\mu_{\mathrm{H}}}{k T}-\exp \frac{\mu_{1}}{k T}\right) t_{1}, \\
& \Delta N_{2}=h_{2}\left(\exp \frac{\mu_{\mathrm{L}}}{k T}-\exp \frac{\mu_{2}}{k T}\right) t_{2}, \\
& \Delta N_{3}=h_{3}\left(\exp \frac{\mu_{3}}{k T}-\exp \frac{\mu_{0}}{k T}\right) t_{3}, \\
& \Delta N_{4}=h_{4}\left(\exp \frac{\mu_{4}}{k T}-\exp \frac{\mu_{\mathrm{M}}}{k T}\right) t_{4},
\end{aligned}
$$

where $k$ is Boltzmann's constant and $T$ is temperature.

\section{Fundamental optimal relation}

The laws of mass and energy conservations give

$$
\begin{aligned}
& \Delta N_{1}+\Delta N_{2}-\Delta N_{3}-\Delta N_{4}=0, \\
& \mu_{1} \Delta N_{1}+\mu_{2} \Delta N_{2}-\mu_{3} \Delta N_{3}-\mu_{4} \Delta N_{4}=0 .
\end{aligned}
$$

Parameter $b$ denotes the ratio of the transferred energy quantity of the second mass reservoir to the total transferred energy quantity of the first and the second mass reservoirs

$$
b=\mu_{\mathrm{L}} \Delta N_{2} /\left(\mu_{\mathrm{H}} \Delta N_{1}+\mu_{\mathrm{L}} \Delta N_{2}\right) .
$$

The COP $\chi$ and the rate of energy pumping $\Sigma$ of the chemical potential transformer are

$$
\chi=\frac{\mu_{0} \Delta N_{3}}{\mu_{\mathrm{H}} \Delta N_{1}+\mu_{\mathrm{L}} \Delta N_{2}}, \quad \Sigma=\frac{\mu_{0} \Delta N_{3}}{\tau} .
$$

Combining Eqs. (2)-(7) gives

$$
\begin{aligned}
\chi & =\mu_{0}\left[(1-b) \mu_{\mathrm{H}}^{-1}\left(\mu_{1}-\mu_{4}\right)+b \mu_{\mathrm{L}}^{-1}\left(\mu_{2}-\mu_{4}\right)\right] \\
& \times\left(\mu_{3}-\mu_{4}\right)^{-1}, \\
\Sigma & =\mu_{0}\left[\frac{(1-b)\left(\mu_{1}-\mu_{4}\right)}{\mu_{\mathrm{H}}}+\frac{b\left(\mu_{2}-\mu_{4}\right)}{\mu_{\mathrm{L}}}\right] \\
& \times\left\{( \mu _ { 3 } - \mu _ { 4 } ) \left[\frac{(1-b) \mu_{\mathrm{H}}^{-1}}{h_{1}\left(\exp \frac{\mu_{\mathrm{H}}}{k T}-\exp \frac{\mu_{1}}{k T}\right)}\right.\right. \\
& +\frac{b \mu_{\mathrm{L}}^{-1}}{h_{2}\left(\exp \frac{\mu_{\mathrm{L}}}{k T}-\exp \frac{\mu_{2}}{k T}\right)} \\
& +\frac{(1-b) \mu_{\mathrm{H}}^{-1}\left(\mu_{1}-\mu_{4}\right)+b \mu_{\mathrm{L}}^{-1}\left(\mu_{2}-\mu_{4}\right)}{h_{3}\left(\exp \frac{\mu_{3}}{k T}-\exp \frac{\mu_{0}}{k T}\right)\left(\mu_{3}-\mu_{4}\right)} \\
& \left.\left.+\frac{(1-b) \mu_{\mathrm{H}}^{-1}\left(\mu_{3}-\mu_{1}\right)+b \mu_{\mathrm{L}}^{-1}\left(\mu_{3}-\mu_{2}\right)}{h_{4}\left(\exp \frac{\mu_{4}}{k T}-\exp \frac{\mu_{\mathrm{M}}}{k T}\right)\left(\mu_{3}-\mu_{4}\right)}\right]\right\}^{-1} .
\end{aligned}
$$

Now, the problem is to determine the optimal rate of energy pumping of the chemical potential transformer for a given COP. Therefore, a Lagrangian function $L=$ $\Sigma+\lambda \chi$ can be introduced, where $\lambda$ is the Lagrangian multiplier. There exists the following relationship:

$$
\begin{aligned}
L & =\mu_{0}\left[(1-b) \mu_{\mathrm{H}}^{-1}\left(\mu_{1}-\mu_{4}\right)+b \mu_{\mathrm{L}}^{-1}\left(\mu_{2}-\mu_{4}\right)\right] \\
& \times\left(\mu_{3}-\mu_{4}\right)^{-1}\left\{\left[\frac{(1-b) \mu_{\mathrm{H}}^{-1}}{h_{1}\left(\exp \frac{\mu_{\mathrm{H}}}{k T}-\exp \frac{\mu_{1}}{k T}\right)}\right.\right. \\
& +\frac{b \mu_{\mathrm{L}}^{-1}}{h_{2}\left(\exp \frac{\mu_{\mathrm{L}}}{k T}-\exp \frac{\mu_{2}}{k T}\right)} \\
& +\frac{(1-b) \mu_{\mathrm{H}}^{-1}\left(\mu_{1}-\mu_{4}\right)+b \mu_{\mathrm{L}}^{-1}\left(\mu_{2}-\mu_{4}\right)}{h_{3}\left(\exp \frac{\mu_{3}}{k T}-\exp \frac{\mu_{0}}{k T}\right)\left(\mu_{3}-\mu_{4}\right)} \\
& \left.\left.+\frac{(1-b) \mu_{\mathrm{H}}^{-1}\left(\mu_{3}-\mu_{1}\right)+b \mu_{\mathrm{L}}^{-1}\left(\mu_{3}-\mu_{2}\right)}{h_{4}\left(\exp \frac{\mu_{4}}{k T}-\exp \frac{\mu_{\mathrm{M}}}{k T}\right)\left(\mu_{3}-\mu_{4}\right)}\right]^{-1}+\lambda\right\} .
\end{aligned}
$$

From the Euler-Lagrange equations $\partial L / \partial \mu_{1}=0$, $\partial L / \partial \mu_{2}=0, \partial L / \partial \mu_{3}=0$ and $\partial L / \partial \mu_{4}=0$, one can find that the following equations must be satisfied:

$$
\begin{aligned}
& \frac{\exp \frac{\mu_{1}}{k T}}{h_{1}\left(\exp \frac{\mu_{\mathrm{H}}}{k T}-\exp \frac{\mu_{1}}{k T}\right)^{2}}=\frac{\exp \frac{\mu_{2}}{k T}}{h_{2}\left(\exp \frac{\mu_{\mathrm{L}}}{k T}-\exp \frac{\mu_{2}}{k T}\right)^{2}} \\
& =\frac{\exp \frac{\mu_{3}}{k T}}{h_{3}\left(\exp \frac{\mu_{3}}{k T}-\exp \frac{\mu_{0}}{k T}\right)^{2}} \\
& =\frac{\exp \frac{\mu_{4}}{k T}}{h_{4}\left(\exp \frac{\mu_{4}}{k T}-\exp \frac{\mu_{\mathrm{M}}}{k T}\right)^{2}} .
\end{aligned}
$$

Substituting Eq. (11) into Eqs. (8) and (9) yields the optimal dimensionless rate of energy pumping $\Sigma^{*}=$ $\Sigma /\left(h_{1} \mu_{\mathrm{H}} \exp \frac{\mu_{\mathrm{H}}}{k T}\right)$ and the COP as follows:

$$
\begin{aligned}
\Sigma^{*} & =\left\{\frac{1}{1-x}+\frac{b_{1} b \mu_{\mathrm{L}}^{-1} \mu_{\mathrm{H}}}{(1-b)\left(\exp \frac{\mu_{\mathrm{L}}}{k T}-\beta\right) / \exp \frac{\mu_{\mathrm{H}}}{k T}}\right. \\
& +b_{2} \frac{\left[\ln \left(x \exp \frac{\mu_{\mathrm{H}}}{k T}\right)-\ln \gamma\right]+b \mu_{\mathrm{L}}^{-1} \mu_{\mathrm{H}}(\ln \alpha-\ln \gamma)}{(\ln \beta-\ln \alpha)\left(\beta-\exp \frac{\mu_{0}}{k T}\right) / \exp \frac{\mu_{\mathrm{H}}}{k T}} \\
& \left.+b_{3} \frac{\left[\ln \beta-\ln \left(x \exp \frac{\mu_{\mathrm{H}}}{k T}\right)\right]+b \mu_{\mathrm{L}}^{-1} \mu_{\mathrm{H}}(\ln \beta-\ln \alpha)}{(\ln \beta-\ln \gamma)\left(\gamma-\exp \frac{\mu_{\mathrm{M}}}{k T}\right) / \exp \frac{\mu_{\mathrm{H}}}{k T}}\right\}^{-1} \\
& \times\left\{\left[\ln \left(x \exp \frac{\mu_{\mathrm{H}}}{k T}\right)-\ln \gamma\right]+b(1-b)^{-1} \mu_{\mathrm{L}}^{-1} \mu_{\mathrm{H}}\right. \\
& \times(\ln \alpha-\ln \gamma)\}(\ln \beta-\ln \gamma)^{-1}, \\
\chi & =(\ln \beta-\ln \gamma)^{-1}\left\{( 1 - b ) \mu _ { 0 } \mu _ { \mathrm { H } } ^ { - 1 } \left[\ln \left(x \exp \frac{\mu_{\mathrm{H}}}{k T}\right)\right.\right. \\
& \left.-\ln \gamma]+b \mu_{0} \mu_{\mathrm{L}}^{-1}(\ln \alpha-\ln \gamma)\right\},
\end{aligned}
$$

where $x=\exp \frac{\mu_{1}}{k T} / \exp \frac{\mu_{\mathrm{H}}}{k T}, b_{1}=h_{1} / h_{2}, b_{2}=h_{1} / h_{3}$, $b_{3}=h_{1} / h_{4}$, 


$$
\begin{aligned}
& \alpha=\exp \frac{\mu_{2}}{k T}=-\sqrt{b_{1} \exp \frac{\mu_{\mathrm{L}}}{k T}(1-x)^{2} x^{-1} \exp \frac{\mu_{\mathrm{H}}}{k T}+\left[\frac{1}{2} b_{1}(1-x)^{2} x^{-1} \exp \frac{\mu_{\mathrm{H}}}{k T}\right]^{2}}+\exp \frac{\mu_{\mathrm{L}}}{k T}+\frac{1}{2} b_{1}(1-x)^{2} x^{-1} \exp \frac{\mu_{\mathrm{H}}}{k T}, \\
& \beta=\exp \frac{\mu_{3}}{k T}=\sqrt{b_{2} \exp \frac{\mu_{0}}{k T}(1-x)^{2} x^{-1} \exp \frac{\mu_{\mathrm{H}}}{k T}+\left[\frac{1}{2} b_{2}(1-x)^{2} x^{-1} \exp \frac{\mu_{\mathrm{H}}}{k T}\right]^{2}}+\exp \frac{\mu_{0}}{k T}+\frac{1}{2} b_{2}(1-x)^{2} x^{-1} \exp \frac{\mu_{\mathrm{H}}}{k T}
\end{aligned}
$$

and

$$
\gamma=\exp \frac{\mu_{4}}{k T}=\sqrt{b_{3} \exp \frac{\mu_{\mathrm{M}}}{k T}(1-x)^{2} x^{-1} \exp \frac{\mu_{\mathrm{H}}}{k T}+\left[\frac{1}{2} b_{3}(1-x)^{2} x^{-1} \exp \frac{\mu_{\mathrm{H}}}{k T}\right]^{2}}+\exp \frac{\mu_{\mathrm{M}}}{k T}+\frac{1}{2} b_{3}(1-x)^{2} x^{-1} \exp \frac{\mu_{\mathrm{H}}}{k T} .
$$

Eliminating $x=\exp \frac{\mu_{1}}{k T} / \exp \frac{\mu_{\mathrm{H}}}{k T}$ from Eqs. (12) and (13) yields the fundamental optimal relation between the dimensionless rate of energy pumping and the COP of an endoreversible four-reservoir chemical potential transformer. It can reveal the $\Sigma-\chi$ characteristics of an endoreversible chemical potential transformer with diffusive mass transfer, as shown in Fig. 3.

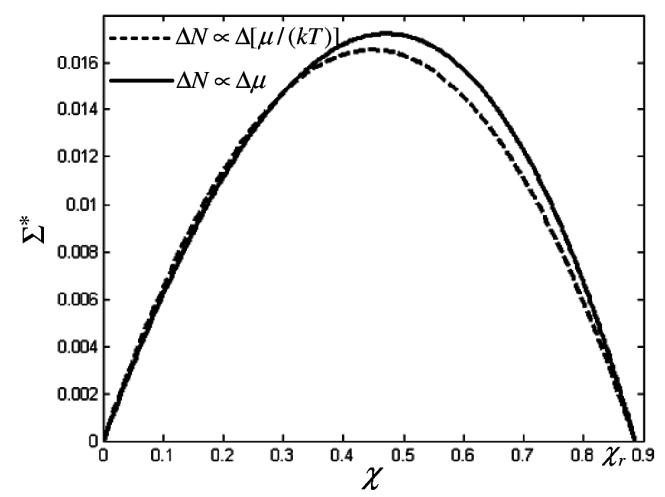

Fig. 3. Relation between the optimal dimensionless rate of energy pumping and COP.

\section{Results and discussion}

\subsection{Numerical examples}

In order to study the characteristics of a four-reservoir endoreversible chemical potential transformer with the diffusive mass transfer law, one numerical example is provided. In the calculations, $b=0.5, b_{1}=1.2, b_{2}=1, b_{3}=$ $1.1, \exp \frac{\mu_{\mathrm{M}}}{k T}=2, \exp \frac{\mu_{\mathrm{L}}}{k T} / \exp \frac{\mu_{\mathrm{M}}}{k T}=e^{2}, \exp \frac{\mu_{\mathrm{H}}}{k T} / \exp \frac{\mu_{\mathrm{M}}}{k T}=$ $e^{3}, \exp \frac{\mu_{0}}{k T} / \exp \frac{\mu_{\mathrm{M}}}{k T}=e^{5}$ and $T=300 \mathrm{~K}$ are set. The numerical values adopted in the calculations were selected. It was not connected to practice. It is for illustrations.

The characteristic curves between the dimensionless rate of energy pumping and the COP of four-reservoir chemical potential transformer with the diffusive mass transfer law are shown in Fig. 3 by solid line. The performance of the four-reservoir chemical potential transformer with linear mass transfer law is also shown in Fig. 3 by dashed line. The influence of $b$ on $\Sigma^{*}$ versus $\chi$ characteristic is shown in Fig. 4. The influence of $\left(\mu_{0}-\mu_{\mathrm{L}}\right) /(k T)$ on $\Sigma^{*}$ versus $\chi$ characteristic with
$\left(\mu_{\mathrm{H}}-\mu_{\mathrm{M}}\right) /(k T)=3$ is shown in Fig. 5. The influence of $\left(\mu_{\mathrm{H}}-\mu_{\mathrm{M}}\right) /(k T)$ on $\Sigma^{*}$ versus $\chi$ characteristic with $\left(\mu_{0}-\mu_{\mathrm{L}}\right) /(k T)=3$ is shown in Fig. 6 .

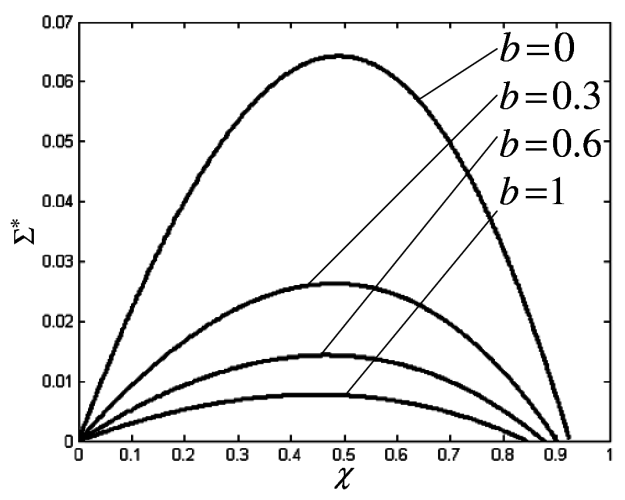

Fig. 4. The influence of $b$ on $\Sigma^{*}$ versus $\chi$ characteristic.

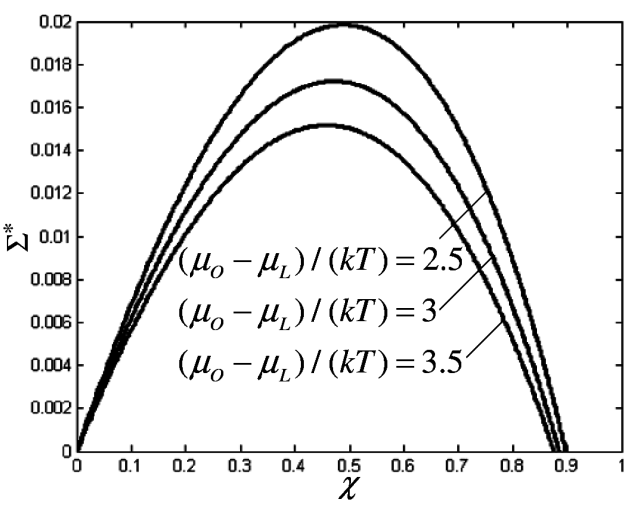

Fig. 5. The influence of $\left(\mu_{0}-\mu_{\mathrm{L}}\right) /(k T)$ on $\Sigma^{*}$ versus $\chi$ characteristic with $\left(\mu_{\mathrm{H}}-\mu_{\mathrm{M}}\right) /(k T)=3$.

It can be seen clearly from Fig. 3 that the characteristic curves between the rate of energy pumping and the COP are parabolic-like ones. With the same value of chemical potentials of the reservoirs, the rate of energy pumping $\Sigma^{*}$ with diffusive mass transfer law is larger than that with linear mass transfer law for the same COP $\chi$. One can have the conclusion that the chemical converters governed by diffusive mass transfer are inherently more 


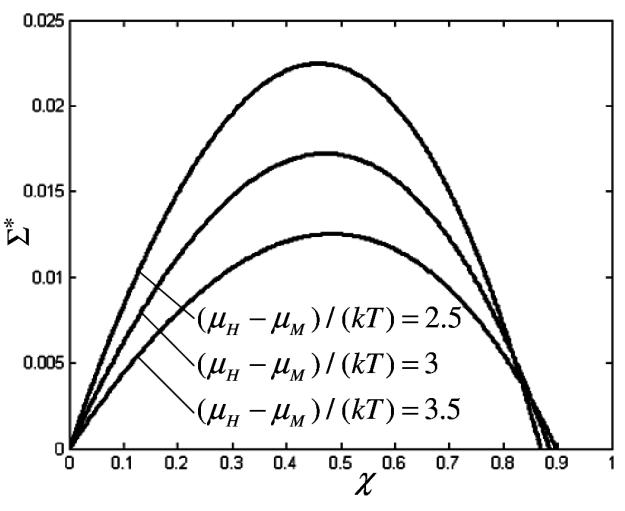

Fig. 6. The influence of $\left(\mu_{\mathrm{H}}-\mu_{\mathrm{M}}\right) /(k T)$ on $\Sigma^{*}$ versus $\chi$ characteristic with $\left(\mu_{0}-\mu_{\mathrm{L}}\right) /(k T)=3$.

efficient than those obeying linear mass transfer, as mentioned by Ref. [12]. When $\Sigma=0$, one can obtain the reversible COP $\chi_{r}$ of the four-reservoir chemical potential transformer, namely

$$
\chi_{r}=\frac{1-\left[(1-b) / \mu_{\mathrm{H}}+b / \mu_{\mathrm{L}}\right] \mu_{\mathrm{M}}}{1-\mu_{0} / \mu_{\mathrm{M}}} .
$$

It can be seen that the optimal COP of the four-reservoir endoreversible chemical potential transformer cannot exceed the reversible COP $\chi_{r}$. This shows that the real chemical potential transformer must decrease the COP level if one wants to obtain some rate of energy pumping. When $\chi<\chi_{r}$, there exists a maximum rate of energy pumping $\Sigma_{\max }^{*}$, and the corresponding COP is $\chi_{\Sigma^{*}}$. $\Sigma_{\max }^{*}$ and $\chi_{\Sigma^{*}}$ are two important parameters of the four-reservoir endoreversible chemical potential transformer, because they determine the upper bound for the rate of energy pumping and the lower bound of the COP, and provide a finite-time thermodynamic criteria for the optimal design of real chemical potential transformer, i.e. the real chemical potential transformer design must match the condition $\chi_{r}>\chi \geq \chi_{\Sigma^{*}}$ to make the chemical potential transformer operate under the optimal conditions.

It can be seen from Fig. 4 that $\Sigma^{*}$ decreases as $b$ increases for the fixed $\chi$.

It can be seen from Fig. 5 that $\Sigma^{*}$ decreases as $\left(\mu_{0}-\right.$ $\left.\mu_{\mathrm{L}}\right) /(k T)$ increases for the fixed $\chi$, while $\Sigma_{\max }^{*}$ and $\chi_{r}$ both decrease as $\left(\mu_{0}-\mu_{\mathrm{L}}\right) /(k T)$ increases.

One can see from Fig. 6 that three curves intersect with each other as $\left(\mu_{\mathrm{H}}-\mu_{\mathrm{M}}\right) /(k T)$ changes, but the points of intersection are close. When $\chi$ is smaller than the values at these points of intersection, $\Sigma^{*}$ increases as $\left(\mu_{\mathrm{H}}-\mu_{\mathrm{M}}\right) /(k T)$ decreases for the same $\chi$. When $\chi$ is larger than the values at these points of intersection, $\Sigma^{*}$ decreases as $\left(\mu_{\mathrm{H}}-\mu_{\mathrm{M}}\right) /(k T)$ decreases for the same $\chi$, and $\Sigma_{\max }^{*}$ decreases as $\left(\mu_{\mathrm{H}}-\mu_{\mathrm{M}}\right) /(k T)$ increases, while both $\chi_{\Sigma^{*}}$ and $\chi_{r}$ increase as $\left(\mu_{\mathrm{H}}-\mu_{\mathrm{M}}\right) /(k T)$ increases.

\subsection{Entropy production rate}

Owing to the irreversibility of mass transfer, the entropy production rate for the cyclic system of four- -reservoir endoreversible chemical potential transformer is larger than zero. If the environment temperature of the cyclic system is represented by $T_{0}$, one can obtain the entropy production rate as follows:

$$
\begin{aligned}
\sigma & =\frac{1}{T_{0} \tau}\left(\mu_{\mathrm{H}} \Delta N_{1}+\mu_{\mathrm{L}} \Delta N_{2}-\mu_{o} \Delta N_{3}-\mu_{\mathrm{M}} \Delta N_{4}\right) \\
& =\frac{\Sigma}{T_{0}}\left\{\frac{\mu_{\mathrm{M}}}{\chi}\left[1-\left(\frac{1-b}{\mu_{\mathrm{H}}}+\frac{b}{\mu_{\mathrm{L}}}\right)\right]-\left(1-\frac{\mu_{\mathrm{M}}}{\mu_{\mathrm{L}}}\right)\right\} .
\end{aligned}
$$

Combining Eqs. (12)-(14), one can obtain the relation between the entropy production rate and the rate of energy pumping or between the entropy production rate and the COP.

\subsection{Applications}

The cyclic model established herein can be applied to the systems such as mass exchangers, electrochemical, photochemical and solid state devices, and the fuel pumps for solar energy conversion systems [37]. It should be pointed out that the related quantities here, in general, have different definition forms for different systems. For example, in solid-state devices, Eq. (2) is referred to as the current-voltage relations, where $\mathrm{d} N / \mathrm{d} t$ is the current, and $\Delta \mu$ is the voltage, while in electrochemical devices, $\Delta \mu$ represents the gradient of electrochemical potential and the conjugated flow is the transport of ions. When the transferred working substance in a chemical potential transformer is electrons or ions, $\Delta N$ is the transferred electric charge, $\Delta \mu$ is electromotive force (EMF) and $\mathrm{h}^{-1}$ is the resistance. Generally, $\mathrm{h}^{-1}$ is the mass flow resistance. Moreover, the working substance in a chemical potential transformer may be gas or liquid molecules in mass exchangers in addition to a current of electrons in solid-state devices [9-11]. The results obtained herein can provide some new theoretical instructions for the optimal design of these devices.

It should be pointed out that the constitutive laws for the four chemical product conductors will be expressed not in terms of the chemical potentials $\mu$ but in terms of the more common concentration $c$. Both variables can be interchanged, according to Eq. (1). Similarly, other variables, such as activity, may be introduced to replace the chemical potential with the help of some relevant relations in thermodynamics [38].

\section{Conclusion}

The performance of the isothermal endoreversible four-reservoir chemical potential transformer with diffusive mass transfer law is analyzed and optimized by using finite-time thermodynamics in this paper. The optimal relation between the rate of energy pumping and the COP of the endoreversible chemical potential transformer is derived. It can be found that the optimal relation of the endoreversible four-reservoir chemical potential transformer with diffusive mass transfer law is similar to the chemical potential transformer with linear mass 
transfer law, but is more efficient than that obeying linear mass transfer. Moreover, the optimally operating regions are also analyzed. The results obtained herein may be used in the design of mass exchangers, electrochemical, photochemical and solid state devices, fuel pumps and so on. This can provide some new theoretical instructions for the optimal design of these devices.

The further assumption by adding irreversibilities due to mass leakage and internal source of irreversibility is necessary. The analysis of chemical potential transformer operating with both temperature and chemical potential differences, and for an arbitrary number of chemical components is also necessary. They will be the subjects of a further presentation.

\section{Acknowledgments}

This paper is supported by the National Natural Science Foundation of P.R. China (project No. 10905093), the Program for New Century Excellent Talents in University of P.R. China (project No. NCET-04-1006) and the Foundation for the Author of National Excellent Doctoral Dissertation of P.R. China (project No. 200136). The authors wish to thank the reviewers for their careful, unbiased and constructive suggestions, which led to this revised manuscript.

\section{References}

[1] B. Andresen, Finite-Time Thermodynamics, Physics Laboratory, University of Copenhagen, Copenhagen 1983.

[2] M. Feidt, Thermodynamique et Optimisation Energetique des Systems et Procedes, 2nd ed., Technique et Documentation, Lavoisier, Paris 1996 (in French).

[3] R.S. Berry, V.A. Kazakov, S. Sieniutycz, Z. Szwast, A.M. Tsirlin, Thermodynamic Optimization of Finite Time Processes, Wiley, Chichester 1999.

[4] P. Salamon, J.D. Nulton, G. Siragusa, T.R. Andresen, A. Limon, Energy, Int. J. 26, 307 (2001).

[5] L. Chen, Finite-Time Thermodynamic Analysis of Irreversible Processes and Cycles, Higher Education Press, Beijing 2005.

[6] A. De Vos, Thermodynamics of Solar Energy Conversion, Wiley-VCH, Berlin 2008.

[7] M. Feidt, Int. J. Exergy 5, 500 (2008).

[8] S. Sieniutycz, J. Jezowski, Energy Optimization in Process Systems, Elsevier, Oxford 2009.

[9] A. De Vos, Solar Cells 31, 181 (1991).

[10] A. De Vos, J. Phys. Chem. 95, 4534 (1991).

[11] A. De Vos, J. Appl. Phys. 74, 3631 (1993).

[12] J.M. Gordon, V.N. Orlov, J. Appl. Phys. 74, 5303 (1993).
[13] L. Chen, F. Sun, C. Wu, Energy Convers. Mgmt. 38, 1841 (1997)

[14] L. Chen, F. Sun, C. Wu, J. Phys. D, Appl. Phys. 31, 1595 (1998).

[15] G. Lin, J. Chen, E. Bruck, Appl. Energy 78, 123 (2004).

[16] G. Lin, J. Chen, Appl. Energy 70, 35 (2001).

[17] G. Lin, J. Chen, E. Brück, B. Hua, Math. Comput. Model. 43, 743 (2006).

[18] G. Lin, J. Chen, B. Hua, Energy Convers Mgmt. 44, 1719 (2003).

[19] S. Wu, S. Zheng, G. Lin, J. Chen, J. Xiamen University 43, 479 (2004) (in Chinese).

[20] S. Wu, G. Lin, J. Chen, C. Wu, Int. J. Ambient Energy 28, 171 (2007).

[21] J. Chen, J. Phys. D, Appl. Phys. 31, 3316 (1998).

[22] X. Qin, L. Chen, F. Sun, C. Wu, Open Syst. Inform. Dyn. 11, 147 (2004).

[23] A. De Vos, Sol. Energy Mater. Sol. Cells 31, 75 (1993).

[24] A. De Vos, Sol. Energy Mater. Sol. Cells 38, 11 (1995).

[25] D. Xia, L. Chen, F. Sun, C. Wu, Appl. Energy 84, 56 (2007).

[26] L. Chen, D. Xia, F. Sun, Proc. I Mech. E, Part C: Mech. Eng. Sci. 222, 1523 (2008).

[27] D. Xia, L. Chen, F. Sun, Appl. Thermal Eng. 27, 1534 (2007).

[28] D. Xia, L. Chen, F. Sun, Proc. I Mech. E, Part C: Mech. Eng. Sci. 222, 689 (2008).

[29] S. Sieniutycz, M. Spakovsky, Energy Convers. Mgmt. 39, 1423 (1998).

[30] S. Sieniutycz, Int. J. Eng. Sci. 36, 577 (1998).

[31] A.M. Tsirlin, V.A. Kazakov, Phys. Rev. E 62, 307 (2000).

[32] L. Chen, D. Xia, F. Sun, Proc. I Mech. E, Part C: Mech. Eng. Sci. 222, 1535 (2008).

[33] D. Xia, L. Chen, F. Sun, Int. J. Sustainable Energy 27, 39 (2008).

[34] D. Xia, L. Chen, F. Sun, C. Wu, Int. J. Ambient Energy 29, 9 (2008).

[35] Å. Jernqvist, K. Abrahamsson, G. Aly, Heat Recovery Syst. CHP 12, 323 (1992).

[36] T. Morosuk, C. Morosuk, M. Feidt, Energy, The Int. J. 29, 2517 (2004).

[37] K. Abrahamsson, Å. Jernqvist, Int. J. Refrig. 16, 240 (1993).

[38] G.J. Van Wylen, R.E. Sonntag, Fundamentals of Chemical Thermodynamics, 2nd ed., Wiley, New York 1973. 